S.M. $\mathrm{CHOl}$

KODAI MATH. J.

15 (1992), 279-295

\title{
3-DIMENSIONAL SPACE-LIKE SUBMANIFOLDS WITH PARALLEL MEAN CURVATURE VECTOR OF AN INDEFINITE SPACE FORM
}

\author{
By SOON MeEn ChOI
}

\section{Introduction.}

Let $M_{p}^{n+p}(c)$ be an $(n+p)$-dimensional connected indefinite Riemannian manifold of index $p$ and of constant curvature $c$, which is called an indefinite space form of index $p$. According to $c>0, c=0$ or $c<0$ it is denoted by $S_{p}^{n+p}(c)$, $\boldsymbol{R}_{p}^{n+p}$ or $H_{p}^{n+p}(c)$. A submanifold $M$ of an indefinite space form $M_{p}^{n+p}(c)$ is said to be space-like if the induced metric on $M$ from that of the ambient space is positive definite. Now it is pointed out by many physicians that space-like hypersurfaces with constant mean curvature of arbitrary spacetimes get interesting in relativity theory. Also, from the differential point of view, an entire space-like hypersurface with constant mean curvature of an indefinite space form are studied by many authors (for examples: [1], [2], [3], [4] and so on). For a complete space-like submanifold $M$ with parallel mean curvature vector of $S_{p}^{n+p}(c)$, it is also seen by Cheng [3] that $M$ is totally umbilic if $n=2$ and $H^{2} \leqq c$ or if $n>2$ and $n^{2} H^{2}<4(n-1) c$, where $H$ denotes the mean curvature, i.e., the norm of the mean curvature vector. On the other hand, Aiyama and Cheng [1] prove recently the following.

THEOREM. Let $M$ be a 3-dimensional complete space-like hypersurface with parallel mean curvature $H$ in a Lorentzian space form $M_{1}^{4}(c)$. If $\sup \operatorname{Ric}(M)$ $<3\left(c-H^{2}\right)$, then $M$ is totally umbilic, and $c>H^{2}$.

The purpose of this paper is to research the similar problem to the above theorem for 3-dimensional complete space-like submanifolds with parallel mean curvature vector of an indefinite space form and to prove the following.

THEOREM 1. Let $M$ be a 3-dimensional complete space-like submanifold with non-zero parallel mean curvature vector $\boldsymbol{h}$ of an indefinite space form $S_{p}^{3+p}(c)$, $p \geqq 2$. If it satisfies

Keywords. indefinite space forms, space-like submanifolds, mean curvature vectors, pseudo-umbilic, isoparametric submanifolds.

Received January 13, 1992. 


$$
\frac{8}{9} c \leqq H^{2} \leqq c \quad \text { and } \quad \operatorname{Ric}(M) \leqq \delta_{1}<3\left(c-H^{2}\right),
$$

then $M$ is totally umbilic.

Let $M$ be a 3-dimensional complete space-like submanifold with non-zero parallel mean curvature vector of an indefinite space form $M_{p}^{3+p}(c)$. We denote by $S$ the square of the length of the second fundamental form of $M$. It is seen in Proposition 3.2 that if $M$ is pseudo-umbilic and if $H^{2}>c$, then it satisfies

$$
S \leqq 3 p H^{2}-3(p-1) c
$$

and also in Remark 3.2 a natural example satisfying the equality of (1.2) is given. Conversely we can prove

THEOREM 2. Let $M$ be a 3-dimensional complete space-like submanifold with non-zero parallel mean curvature vector $\boldsymbol{h}$ of an indefinite space form $M_{p}^{3+p}(c), c$ $\leqq 0, p \geqq 2$. If it satisfies

$$
\operatorname{Ric}(M) \leqq \delta_{1}<\frac{3}{2}(p-3)\left(H^{2}-c\right) \text { and } S \geqq 3 p H^{2}-3(p-1) c,
$$

then the following assertions hold,

(1) $c<0$, and $p \geqq 4$,

(2) $M$ is congruent to

$$
\begin{aligned}
& \text { 1. } H^{2}\left(c_{1}\right) \times H^{1}\left(c_{2}\right), \quad p=4, \\
& \text { 2. } H^{1}\left(c_{1}\right) \times H^{1}\left(c_{2}\right) \times H^{1}\left(c_{3}\right), \quad p=4,5,
\end{aligned}
$$

where $H^{n}(c)$ denotes an n-dimensional hyperbolic space of constant curvature $c$.

\section{Preliminaries.}

Throughout this paper all manifolds are assumed to be smooth, connected without boundary. We discuss in smooth category. Let $M_{p}^{n+p}(c)$ be an $(n+p)$ dimensional indefinite Riemannian manifold of constant curvature $c$ whose index is $p$, which is called an indefinite space form of constant curvature $c$ and with index $p$. Let $M$ be an $n$-dimensional submanifold of an $(n+p)$-dimensional indefinite space form $M_{p}^{n+p}(c)$ of index $p>0$. The submanifold $M$ is said to be space-like if the induced metric on $M$ from that of the ambient space is positive definite. We choose a local field of orthonormal frames $e_{1}, \cdots, e_{n+p}$ adapted to the indefinite Riemannian metric of $M_{p}^{n+p}(c)$ and the dual coframe $\omega_{1}, \cdots, \omega_{n+p}$ in such a way that, restricted to the submanifold $M, e_{1}, \cdots, e_{n}$ are tangent to $M$. Then connection forms $\left\{\omega_{A B}\right\}$ of $M_{p}^{n+p}(c)$ are characterized by the structure equations 


$$
\begin{aligned}
& \left\{\begin{array}{l}
d \omega_{A}+\sum \varepsilon_{B} \omega_{A B} \wedge \omega_{B}=0, \quad \omega_{A B}+\omega_{B A}=0, \\
d \omega_{A B}+\sum \varepsilon_{C} \omega_{A C} \wedge \omega_{C B}=\Omega_{A B}, \\
\Omega_{A B}=-\frac{1}{2} \sum \varepsilon_{C} \varepsilon_{D} R_{A B C D}^{\prime} \omega_{C} \wedge \omega_{D},
\end{array}\right. \\
& R_{A B C D}^{\prime}=c \varepsilon_{A} \varepsilon_{B}\left(\delta_{A D} \delta_{B C}-\delta_{A C} \delta_{B D}\right),
\end{aligned}
$$

where $\Omega_{A B}$ (resp. $R_{A B C D}^{\prime}$ ) denotes the indefinite Riemannian curvature form (resp. the components of the indefinite Riemannian curvature tensor) of $M_{p}^{n+p}(c)$. Therefore the components of the Ricci curvature tensor $R i c^{\prime}$ and the scalar curvature $r^{\prime}$ of $M_{p}^{n+p}(c)$ are given as

$$
R_{A B}^{\prime}=c(n+p-1) \varepsilon_{A} \delta_{A B}, \quad r^{\prime}=(n+p)(n+p-1) c .
$$

In the sequel, the following convention on the range of indices is used, unless otherwised stated :

$$
1 \leqq A, B, \cdots \leqq n+p ; 1 \leqq i, j, \cdots \leqq n ; n+1 \leqq \alpha, \beta, \cdots \leqq n+p .
$$

We agree that the repeated indices under a summation sign without indication are summed over the respective range. The canonical forms $\left\{\omega_{A}\right\}$ and the connection forms $\left\{\omega_{A B}\right\}$ restricted to $M$ are also denoted by the same symbols. We then have

$$
\omega_{\alpha}=0 \quad \text { for } \quad \alpha=n+1, \cdots, n+p \text {. }
$$

We see that $e_{1}, \cdots, e_{n}$ is a local field of orthonormal frames adapted to the induced Riemannian metric on $M$ and $\omega_{1}, \cdots, \omega_{n}$ is a local field of its dual coframes on $M$. It follows from (2.1), (2.3) and Cartan's Lemma that

$$
\omega_{\alpha \imath}=\sum h_{\imath j}^{\alpha} \omega_{j}, \quad h_{\imath \jmath}^{\alpha}=h_{j i}^{\alpha} .
$$

The second fundamental form $\alpha$ and the mean curvature vector $\boldsymbol{h}$ of $M$ are defined by

$$
\alpha=-\sum h_{i j}^{\alpha} \omega_{i} \omega_{j} e_{\alpha}, \quad \boldsymbol{h}=-\frac{1}{n} \sum\left(\sum_{i} h_{i i}^{\alpha}\right) e_{\alpha} .
$$

The mean curvature $H$ is defined by

$$
H=|\boldsymbol{h}|=\frac{1}{n} \sqrt{\sum_{i}\left(\sum_{i} h_{i i}^{\alpha}\right)^{2}} .
$$

Let $S=\Sigma\left(h_{i j}^{\alpha}\right)^{2}$ denote the squared norm of the second fundamental form $\alpha$ of $M$. The connection forms $\left\{\omega_{i j}\right\}$ of $M$ are characterized by the structure equations

$$
\left\{\begin{array}{l}
d \omega_{i}+\sum \omega_{i \jmath} \wedge \omega_{j}=0, \quad \omega_{i j}+\omega_{j i}=0 \\
d \omega_{i j}+\sum \omega_{i k} \wedge \omega_{k \jmath}=\Omega_{\imath \jmath}, \\
\Omega_{\imath \jmath}=-\frac{1}{2} \sum R_{\imath j k l} \omega_{k} \wedge \omega_{l}
\end{array}\right.
$$


where $\Omega_{\imath \jmath}$ (resp. $R_{\imath j k l}$ ) denotes the Riemannian curvature form (resp. the components of the Riemannian curvature tensor) of $M$. Therefore, from (2.1) and (2.6), the Gauss equation is given by

$$
R_{\imath j k l}=c\left(\delta_{i l} \delta_{j k}-\delta_{i k} \delta_{j l}\right)-\sum\left(h_{i l}^{\alpha} h_{j k}^{\alpha}-h_{i k}^{\alpha} h_{j l}^{\alpha}\right) .
$$

The components of the Ricci curvature Ric and the scalar curvature $r$ are given by

$$
\begin{gathered}
R_{j k}=(n-1) c \delta_{j k}-\sum h_{i i}^{\alpha} h_{j k}^{\alpha}+\sum h_{i k}^{\alpha} h_{\imath \jmath}^{\alpha}, \\
r=n(n-1) c-n^{2} H^{2}+\Sigma\left(h_{i j}^{\alpha}\right)^{2} .
\end{gathered}
$$

We also have

$$
d \omega_{\alpha \beta}-\Sigma \omega_{\alpha \gamma} \wedge \omega_{\gamma \beta}=-\frac{1}{2} \Sigma R_{\alpha \beta \imath j} \omega_{i} \wedge \omega_{j},
$$

where

$$
R_{\alpha \beta \imath \jmath}=-\Sigma\left(h_{i l}^{\alpha} h_{j l}^{\beta}-h_{\jmath l}^{\alpha} h_{i l}^{\beta}\right) .
$$

The Codazzi equation and the Ricci formula for the second fundamental form are given by

$$
\begin{gathered}
h_{i j k}^{\alpha}-h_{i k j}^{\alpha}=0, \\
h_{i j k l}^{\alpha}-h_{i j l k}^{\alpha}=-\sum h_{i m}^{\alpha} R_{m j k l}-\sum h_{m j}^{\alpha} R_{m i k l}+\sum h_{i j}^{\beta} R_{\beta \alpha k l},
\end{gathered}
$$

where $h_{\imath j k}^{\alpha}$ and $h_{i j k l}^{\alpha}$ denote the components of the covariant differentials $\nabla \alpha$ and $\nabla^{2} \alpha$ of the second fundamental form respectively. The Laplacian $\Delta h_{\imath \jmath}^{\alpha}$ of the components $h_{\imath \jmath}^{\alpha}$ of the second fundamental form $\alpha$ is given by

$$
\Delta h_{\imath j}^{\alpha}=\sum_{k} h_{\imath j k k}^{\alpha} .
$$

From (2.12) we get

$$
\Delta h_{\imath \jmath}^{\alpha}=\sum_{k} h_{k k \imath j}^{\alpha}-\sum h_{k m}^{\alpha} R_{m \imath j k}-\sum h_{m \imath}^{\alpha} R_{m k j k}+\sum h_{k \imath}^{\beta} R_{\beta \alpha j k} .
$$

The following generalized maximum principle due to Omori [8] and Yau [11] will play an important role in this paper.

THEOREM 2.1. Let $M$ be an n-dimensional complete Riemannian manifold whose Rucci curvature is bounded from below. Let $F$ be a $C^{2}$-function bounded from below on $M$, then for any $\varepsilon>0$, there exists a point $p$ in $M$ such that

$$
F(p)<\inf F+\varepsilon, \quad|\operatorname{grad} F|(p)<\varepsilon, \quad \Delta F(p)>-\varepsilon .
$$

By applying this principle the following theorem due to Nishikawa [7] is proved.

THEOREM 2.2. Let $M$ be an n-dimensional complete Riemannian manifold 
whose Ricci curvature is bounded from below. Let $F$ be a non-negative $C^{2}$-function on $M$. If it satisfies

$$
\Delta F \geqq k F^{2},
$$

then $F=0$ on $M$, where $k$ is a positive constant.

\section{Pseudo-umbilic submanifolds.}

This section is concerned with pseudo-umbilic space-like submanifolds of an indefinite space form $M_{p}^{n+p}(c)$. Let $M$ be an $n$-dimensional space-like submanifold with parallel mean curvature vector $\boldsymbol{h} \neq 0$ of $M_{p}^{n+p}(c)$. Because the mean curvature vector is parallel, the mean curvature is constant. We choose $e_{n+1}$ in such a way that its direction coincides with that of the mean curvature vector. Then it is easily seen that we have

$$
\begin{aligned}
& \omega_{\alpha n+1}=0, \quad H=\text { constant }, \\
& H^{\alpha} H^{n+1}=H^{n+1} H^{\alpha}, \\
& \operatorname{tr} H^{n+1}=n H, \quad \operatorname{tr} H^{\alpha}=0
\end{aligned}
$$

for any $\alpha \neq n+1$, where $H^{\alpha}$ denotes an $n \times n$ symmetric matrix $\left(h_{\imath j}^{\alpha}\right)$.

A submanifold $M$ is said to be pseudo-umbilic, if it is umbilic with respect to the direction of the mean curvature vector $\boldsymbol{h}$, that is,

$$
h_{i, j}^{n+1}=H \delta_{i j} .
$$

We denote by $\mu$ an $n \times n$ symmetric matrix with components defined by $\mu_{\imath \jmath}=$ $h_{\imath \jmath}^{n+1}-H \delta_{i \jmath}$. We then have

$$
\operatorname{tr} \mu=0, \quad|\mu|^{2}=\operatorname{tr}(\mu)^{2}=\sum \mu_{\imath \jmath}^{2}=\operatorname{tr}\left(H^{n+1}\right)^{2}-n H^{2} .
$$

So the pseudo-umbilic submanifolds are characterized by the property $\mu=0$. A non-negative function $\tau$ is denoted by $\tau^{2}=\sum_{\beta \neq n+1}\left(h_{i j}^{\beta}\right)^{2}$. Then we have

$$
S=|\mu|^{2}+\tau^{2}+n H^{2},
$$

which means that $S \geqq n H^{2}$, where the equality holds at a point if and only if the point is umbilic. Hence it is seen that $|\mu|^{2}$ as well as $\tau^{2}$ are independent of the choice of the frame fields and they are functions globally defined on $M$. It is also seen that if the pseudo-umbilic submanifold satisfies $\tau=0$, then it is totally umbilic.

Now, in general, it is asserted by Cheng [3] that a complete $n(\geqq 3)$-dimensional space-like submanifold with parallel mean curvature vector $\boldsymbol{h}$ of $S_{p}^{n+p}(c)$ is totally umbilic if it satisfies

$$
H^{2}<\frac{4(n-1)}{n^{2}} c .
$$


PROPOSITION 3.1. Let $M$ be an n-dimensional complete space-like submanifold with non-zero parallel mean curvature vector of $S_{p}^{n+p}(c), p \geqq 2$. If $M$ is pseudoumbilic and if it satisfies

$$
\frac{4(n-1)}{n^{2}} c \leqq H^{2} \leqq c
$$

then $M$ is totally umbilic.

Proof. From (2.13) and the Gauss equation (2.7) and (2.10) we get

$$
\begin{aligned}
\Delta h_{i \jmath}^{\alpha}= & n c h_{i j}^{\alpha}-c \sum h_{k k}^{\alpha} \delta_{i j}+\sum h_{k m}^{\alpha} h_{m k}^{\beta} h_{i j}^{\beta}-2 \sum h_{i k}^{\beta} h_{k m}^{\alpha} h_{m \jmath}^{\beta} \\
& +\sum h_{i m}^{\alpha} h_{m k}^{\beta} h_{k j}^{\beta}-\sum h_{k k}^{\beta} h_{i m}^{\alpha} h_{m j}^{\beta}+\sum h_{i k}^{\beta} h_{m j}^{\alpha} h_{m k}^{\beta}
\end{aligned}
$$

for any index $\alpha$. Moreover we see

$$
\frac{1}{2} \Delta \tau^{2}=\sum_{\alpha \neq n+1}\left(h_{i j k}^{\alpha}\right)^{2}+\sum_{\alpha \neq n+1} h_{i j}^{\alpha} \Delta h_{i j}^{\alpha} .
$$

Accordingly it follows from (3.8) and the above equation that we get

$$
\begin{aligned}
\frac{1}{2} \Delta \tau^{2}= & \sum_{\alpha \neq n+1}\left(h_{i j k}^{\alpha}\right)^{2}+n c \tau^{2}+\sum_{\alpha \neq n+1} h_{k m}^{\alpha} h_{m k}^{\beta} h_{i j}^{\beta} h_{\imath \jmath}^{\alpha} \\
& -2 \sum_{\alpha \neq n+1} h_{i k}^{\beta} h_{k m}^{\alpha} h_{m j}^{\beta} h_{\imath j}^{\alpha}+\sum_{\alpha \neq n+1} h_{i m}^{\alpha} h_{m k}^{\beta} h_{k j}^{\beta} h_{\imath \jmath}^{\alpha} \\
& -n H \sum_{\alpha \neq n+1} h_{i m}^{\alpha} h_{m j}^{n+1} h_{\imath j}^{\alpha}+\sum_{\alpha \neq n+1} h_{i k}^{\beta} h_{k m}^{\beta} h_{m j}^{\alpha} h_{\imath \jmath}^{\alpha},
\end{aligned}
$$

and hence we obtain

$$
\begin{aligned}
\frac{1}{2} \Delta \tau^{2}= & \sum_{\alpha \neq n+1}\left(h_{i j k}^{\alpha}\right)^{2}+n c \tau^{2} \\
& +\sum_{\alpha, \beta \neq n+1} h_{k m}^{\alpha} h_{m k}^{\beta} h_{i j}^{\beta} h_{i j}^{\alpha}-2 \sum_{\alpha, \beta \neq n+1} h_{i k}^{\beta} h_{k m}^{\alpha} h_{m j}^{\beta} h_{i j}^{\alpha} \\
& +\sum_{\alpha, \beta \neq n+1} h_{\imath m}^{\alpha} h_{m k}^{\beta} h_{k j}^{\beta} h_{i j}^{\alpha}+\sum_{\alpha, \beta \neq n+1} h_{i k}^{\beta} h_{k m}^{\beta} h_{m j}^{\alpha} h_{i j}^{\alpha} \\
& +\sum_{\alpha \neq n+1} h_{k m}^{\alpha} h_{m k}^{n+1} h_{i j}^{n+1} h_{i j}^{\alpha}-2 \sum_{\alpha \neq n+1} h_{i k}^{n+1} h_{k m}^{\alpha} h_{m j}^{n+1} h_{i j}^{\alpha} \\
& +\sum_{\alpha \neq n+1} h_{i m}^{\alpha} h_{m k}^{n+1} h_{k j}^{n+1} h_{i j}^{\alpha}-n H \sum_{\alpha \neq n+1} h_{\imath m}^{\alpha} h_{m j}^{n+1} h_{i j}^{\alpha} \\
& +\sum_{\alpha \neq n+1} h_{j m}^{\alpha} h_{m k}^{n+1} h_{k i}^{n+1} h_{i j}^{\alpha} .
\end{aligned}
$$

We put $S_{\alpha \beta}=\sum h_{i j}^{\alpha} h_{i j}^{\beta}$ for any $\alpha, \beta \neq n+1$. Then $\left(S_{\alpha \beta}\right)$ is a $(p-1) \times(p-1)$ symmetric matrix. It can be assumed to be diagonal for a suitable choice of $e_{n+2}, \cdots, e_{n+p}$. Set $S_{\alpha}=S_{\alpha \alpha}$. We then have $\tau^{2}=\Sigma S_{\alpha}$. In general, for a matrix $A=\left(a_{\imath \jmath}\right)$, we define $N(A)=\operatorname{tr}\left(A^{t} A\right)$. Then the above equation can be reduced to 


$$
\begin{gathered}
\frac{1}{2} \Delta \tau^{2}=\sum_{\alpha \neq n+1}\left(h_{i j k}^{\alpha}\right)^{2}+\sum_{\alpha, \beta \neq n+1}\left\{\left(S_{\alpha \beta}\right)^{2}-2 \operatorname{tr} H^{\alpha} H^{\beta} H^{\alpha} H^{\beta}+2 \operatorname{tr} H^{\alpha} H^{\alpha} H^{\beta} H^{\beta}\right\} \\
+\sum_{\alpha \neq n+1}\left\{\sum h_{k m}^{\alpha} h_{m k}^{n+1} h_{\imath \jmath}^{n+1} h_{i j}^{\alpha}-2 \operatorname{tr} H^{\alpha} H^{n+1} H^{\alpha} H^{n+1}\right. \\
\left.+2 \operatorname{tr} H^{\alpha} H^{\alpha} H^{n+1} H^{n+1}-n H \operatorname{tr} H^{\alpha} H^{n+1} H^{\alpha}\right\}
\end{gathered}
$$

By (3.2), (3.3) and (3.4) and the definition of the function $\tau$, we have

$$
\begin{aligned}
\frac{1}{2} \Delta \tau^{2}= & \sum_{\alpha \neq n+1}\left(h_{i j k}^{\alpha}\right)^{2}+n c \tau^{2}+\sum_{\alpha \neq n+1}\left(S_{\alpha}\right)^{2} \\
& +\sum_{\alpha, \beta \neq n+1} N\left(H^{\alpha} H^{\beta}-H^{\beta} H^{\alpha}\right)-n H^{2} \tau^{2} .
\end{aligned}
$$

Obviously we see

$$
\sum_{\alpha, \beta \neq n+1} N\left(H^{\alpha} H^{\beta}-H^{\beta} H^{\alpha}\right) \geqq 0
$$

Suppose $p \geqq 2$. Let

$$
\begin{gathered}
(p-1) \sigma_{1}=\tau^{2}=\sum S_{\alpha}, \\
(p-1)(p-2) \sigma_{2}=2 \sum_{\alpha<\beta, \alpha, \beta \neq n+1} S_{\alpha} S_{\beta} .
\end{gathered}
$$

Then we have

$$
\begin{gathered}
\sum S_{\alpha}^{2}=(p-1) \sigma_{1}^{2}+(p-1)(p-2)\left(\sigma_{1}^{2}-\sigma_{2}\right), \\
\sum_{\alpha<\beta, \alpha, \beta \neq n+1}\left(S_{\alpha}-S_{\beta}\right)^{2}=(p-1)^{2}(p-2)\left(\sigma_{1}^{2}-\sigma_{2}\right) .
\end{gathered}
$$

Hence we obtain

$$
\sum_{\alpha \neq n+1}\left(S_{\alpha}\right)^{2} \geqq(p-1) \sigma_{1}^{2}=\frac{1}{p-1} \tau^{4} .
$$

Accordingly it follows from (3.10), (3.11) and (3.12) that we have

$$
\begin{aligned}
\frac{1}{2} \Delta \tau^{2} & \geqq n c \tau^{2}+\frac{1}{p-1} \tau^{4}-n H^{2} \tau^{2} \\
& =\frac{1}{p-1} \tau^{2}\left\{\tau^{2}-n(p-1)\left(H^{2}-c\right)\right\}
\end{aligned}
$$

By the assumption of the proposition we get

$$
\Delta \tau^{2} \geqq \frac{2}{p-1} \tau^{4}
$$

By (2.8), (3.2) and (3.4) the Ricci curvature is bounded from below by a constant $-(n-1)\left(H^{2}-c\right)$, we can apply Theorem 2.2 to the non-negative function $\tau^{2}$ and we get

Thus $M$ is totally umbilic.

$$
\tau^{2}=0
$$


Remark 3.1. Proposition 3.1 is essentially proved by Cheng [3].

Next the case of $H^{2}>c$ is investigated.

Proposition 3.2. Let $M$ be an n-dimensional complete space-like submanifold with non-zero parallel mean curvature vector of $M_{p}^{n+p}(c), p \geqq 2$. If $M$ is pseudoumbilic and if $H^{2}>c$, then it satisfies

$$
n H^{2} \leqq S \leqq n p H^{2}-n(p-1) c .
$$

Proof. Since $M$ is pseudo-umbilic by the assumption, we have $\mu=0$, which implies $S=\tau^{2}+n H^{2}$ by (3.6). This means that

$$
\begin{aligned}
\tau^{2}-n(p-1)\left(H^{2}-c\right) & =S-n H^{2}-n(p-1)\left(H^{2}-c\right) \\
& =S+n(p-1) c-n p H^{2} .
\end{aligned}
$$

By (3.13) we have

$$
\frac{1}{2} \Delta S \geqq \frac{1}{p-1}\left(S-n H^{2}\right)\left\{S+n(p-1) c-n p H^{2}\right\} .
$$

Given any positive number $a$, a function $F$ is defined by $F=1 / \sqrt{S+a}$, which is bounded from above by $1 / \sqrt{a}$ and is bounded from below by 0 . Since the Ricci curvature of $M$ is bounded from below and since $M$ is complete and space-like, we can apply the Generalized Maximum Principle (Theorem 2.1) to the function $F$. For any given positive number $\varepsilon>0$, there exists a point $p$ at which $F$ satisfies

$$
\inf F>F(p)-\varepsilon, \quad|\operatorname{grad} F|(p)<\varepsilon, \quad \Delta F(p)>-\varepsilon .
$$

Consequently the following relationship

$$
\frac{1}{2} F(p)^{4} \Delta S(p)<3 \varepsilon^{2}+F(p) \varepsilon
$$

can be derived by the simple and direct calculations. For a convergent sequence $\left\{\varepsilon_{m}\right\}$ such that $\varepsilon_{m} \rightarrow 0(m \rightarrow \infty)$ and $\varepsilon<0$, there exists a point sequence $\left\{p_{m}\right\}$ such that $\left\{F\left(p_{m}\right)\right\}$ converges to $F_{0}=\inf F$ by (3.16). On the other hand, it follows from (3.17) that we have

$$
\frac{1}{2} F\left(p_{m}\right)^{4} \Delta S\left(p_{m}\right)<3 \varepsilon_{m}^{2}+F\left(p_{m}\right) \varepsilon_{m} .
$$

The right hand side of (3.18) converges to 0 because $F$ is bounded. Accordingly, for any positive number $\varepsilon(<2)$ there exists a sufficiently large integer $m_{0}$ for which we have

$$
F\left(p_{m}\right)^{4} \Delta S\left(p_{m}\right)<\frac{\varepsilon}{p-1} \quad \text { for } m>m_{0} .
$$

This inequality and (3.15) yield 


$$
2\left\{S\left(p_{m}\right)-n H^{2}\right\}\left\{S\left(p_{m}\right)+n(p-1) c-n p H^{2}\right\}<\left\{S\left(p_{m}\right)+a\right\}^{2} \varepsilon,
$$

and hence we get

$$
\begin{gathered}
(2-\varepsilon) S^{2}\left(p_{m}\right)+2\left\{n(p-1) c-n(p+1) H^{2}-a \varepsilon\right\} S\left(p_{m}\right) \\
-2 n H^{2}\left\{n(p-1) c-n p H^{2}\right\}-a^{2} \varepsilon<0,
\end{gathered}
$$

which implies that the sequence $\left\{S\left(p_{m}\right)\right\}$ is bounded. Thus the infimum $F_{0}$ of $F$ satisfies $F_{0} \neq 0$ by the definition of $F$ and hence the inequality (3.18) implies that $\lim \sup \Delta S\left(p_{m}\right) \leqq 0$. This means that the supremum sup $S$ of the squared norm $S$ satisfies

$$
n H^{2} \leqq \sup S \leqq n p H^{2}-n(p-1) c .
$$

Remark 3.2. Let $M$ be a maximal space-like submanifold of $H_{p \rightarrow 1}^{n+p-1}\left(c^{\prime}\right)$ and let $H_{p-1}^{n+p-1}\left(c^{\prime}\right)$ be a totally umbilic hypersurface of $H_{p}^{n+p}(c)\left(0>c>c^{\prime}\right)$, whose mean curvature is denoted by $H$. Then $M$ can be regarded as a submanifold of $H_{p}^{n+p}(c)$. It is a pseudo-umbilic submanifold with non-zero parallel mean curvature vector $\boldsymbol{h}$ and the squared norm $S$ is given by $S=S^{\prime}+n H^{2}$, where $S^{\prime}$ is denoted the squared norm of $M$ in $H_{p-1}^{n+p-1}\left(c^{\prime}\right)$. According to Proposition 3.2, we have $S \leqq n p H^{2}-n(p-1) c$ in $H_{p}^{n+p}(c)$. The last equality $S=n p H^{2}-n(p-1) c$ is equivalent to $S^{\prime}=n(p-1)\left(H^{2}-c\right)$. This is the second estimation of $S^{\prime}$ obtained by Ishihara [5].

\section{3-dimensional space-like submanifolds.}

In this section, for a 3-dimensional space-like submanifold $M$ we shall give a sufficient condition for $M$ to be pseudo-umbilical. Let $M$ be a 3-dimensional complete space-like submanifold with non-zero parallel mean curvature vector of $M_{p}^{3+p}(c)$. From (2.13) we have

$$
\Delta h_{i \jmath}^{\alpha}=-\sum h_{k m}^{\alpha} R_{m \imath j k}-\sum h_{m \imath}^{\alpha} R_{m k j k}+\sum h_{i k}^{\beta} R_{\beta \alpha j k}
$$

for any indices $\alpha, \imath$ and $\jmath$. By the similar discussion to that in Section 3 we choose $e_{4}$ in such a way that its direction coincides with that of the mean curvature vector. Furthermore, for any fixed point $p$ in $M$ we choose also a local frame field $e_{1}, e_{2}, e_{3}$ such that

$$
h_{i \jmath}^{4}=\lambda_{i} \delta_{i \jmath}
$$

for any $\imath$ and $\jmath$. By (4.1) we have

$$
\frac{1}{2} \Delta|\mu|^{2} \geqq \sum\left(h_{\imath j k}^{4}\right)^{2}-\sum h_{i j}^{4}\left(h_{k m}^{4} R_{m \imath j k}+h_{m \imath}^{4} R_{m k j k}\right),
$$

from which combining with (4.2) it follows that

$$
\frac{1}{2} \Delta|\mu|^{2} \geqq \Sigma\left(h_{i j k}^{4}\right)^{2}+\frac{1}{2} \sum\left(\lambda_{i}-\lambda_{j}\right)^{2} R_{\imath j j i}
$$


On the other hand, since $M$ is a 3-dimensional submanifold, its Weyl conformal curvature tensor vanishes identically on $M$, i.e.,

$$
R_{i j k l}=R_{i l} \delta_{j k}-R_{i k} \delta_{j l}+\delta_{i l} R_{j k}-\delta_{i k} R_{j l}-\frac{r}{2}\left(\delta_{i l} \delta_{j k}-\delta_{i k} \delta_{j l}\right) .
$$

Hence we get

$$
R_{\imath j j i}=R_{i i}+R_{j j}-\frac{r}{2}
$$

for any distinct indices. By $R_{11}+R_{22}+R_{33}=r$, we have

$$
R_{\imath j j i}=\frac{r}{2}-R_{k k}
$$

for any distinct indices. Thus the following equation

$$
\frac{1}{2} \Delta|\mu|^{2} \geqq \Sigma\left(h_{i j k}^{4}\right)^{2}+\frac{1}{2} \Sigma\left(\frac{r}{2}-R_{k k}\right)\left(\lambda_{i}-\lambda_{\jmath}\right)^{2}
$$

is derived.

PROPOSITION 4.1. Let $M$ be a 3-dimensional complete space-like submanifold with non-zero parallel mean curvature vector of $M_{p}^{s+p}(c)$. If it satisfies

$$
\operatorname{Ric}(M) \leqq \delta_{1}<3\left(c-H^{2}\right),
$$

then $M$ is pseudo-umbilic.

Proof. In order to prove this property it suffices to show that the function $|\mu|^{2}$ vanishes identically. By (4.4) and (4.5) we have

$$
\frac{1}{2} \Delta|\mu|^{2} \geqq \frac{1}{4} \Sigma\left(r-2 \delta_{1}\right)\left(\lambda_{i}-\lambda_{j}\right)^{2},
$$

which is equivalent to

$$
\Delta|\mu|^{2} \geqq 3\left(r-2 \delta_{1}\right)|\mu|^{2}
$$

From (2.9) we have

$$
\Delta|\mu|^{2} \geqq 3|\mu|^{2}\left\{|\mu|^{2}+6\left(c-H^{2}\right)-2 \delta_{1}\right\},
$$

from which together with the assumption we have

$$
\Delta|\mu|^{2} \geqq 3|\mu|^{4} \text {. }
$$

Since the Ricci curvature of $M$ is bounded from below and $M$ is complete and space-like and moreover since the function $|\mu|^{2}$ is smooth, Theorem 2.2 yields $|\mu|^{2}=0$, which means that $M$ is pseudo-umbilic.

Remark 4.1. Proposition 4.1 is a higher codimensional version of a theorem 
due to Aiyama and Cheng [1] for a space-like hypersurface.

Proof of Theorem 1. Since the assumption of Theorem 1 satisfies (4.5), $M$ is pseudo-umbilic by Proposition 4.1. Accordingly we can apply Proposition 3.1 to this case and we see that $M$ is totally umbilic.

Next we consider the case of $H^{2}>c$.

Proposition 4.2. Let $M$ be a 3-dimensional complete space-like submanifold with non-zero parallel mean curvature vector of $M_{p}^{3+p}(c)$. If it satisfies $H^{2}>c$ and if

$$
\operatorname{Ric}(M) \leqq \delta_{1}<\frac{3}{2}(p-3)\left(H^{2}-c\right)
$$

then we get

$$
|\mu|^{2} \leqq 3(p-1)\left(H^{2}-c\right) .
$$

Proof. From (2.9) the scalar curvature $r$ is given by $r=6 c-9 H^{2}+S$ and hence we get by (3.6) and (4.7)

$$
\begin{aligned}
r-2 \delta_{1} & >|\mu|^{2}+\tau^{2}-6\left(H^{2}-c\right)-3(p-3)\left(H^{2}-c\right) \\
& \geqq|\mu|^{2}-3(p-1)\left(H^{2}-c\right) .
\end{aligned}
$$

Accordingly (4.6) and the above inequality yield

$$
\Delta|\mu|^{2} \geqq 3|\mu|^{2}\left\{|\mu|^{2}-3(p-1)\left(H^{2}-c\right)\right\} .
$$

Given any positive number $a$, a function $F$ is defined by $1 / \sqrt{|\mu|^{2}+a}$. Then, by the similar method to that in the proof of Proposition 3.2, we obtain the conclusion.

\section{Proof of Theorem 2 .}

In this section Theorem 2 is proved. Let $M$ be an $n(=3)$-dimensional complete space-like submanifold with non-zero parallel mean curvature vector of $M_{p}^{n+p}(c), p \geqq 2$. We assume $H^{2} \geqq c$ and

$$
R \imath c(M) \leqq \delta_{1}<\frac{3}{2}(p-3)\left(H^{2}-c\right) \text { and } S \geqq 3 p H^{2}-3(p-1) c .
$$

Then the scalar curvature $r$ is given by $r=3(p-3)\left(H^{2}-c\right)$ and hence

$$
r-2 \delta_{1} \geqq 3(p-3)\left(H^{2}-c\right)-2 \delta_{1}=\delta
$$

is a positive constant. From (4.6) we have

$$
\Delta|\mu|^{2} \geqq 3 \delta|\mu|^{2} .
$$


Given any positive number $a$, a function $F$ is defined by $F=1 / \sqrt{|\mu|^{2}+a}$, which is bounded from above by $1 / \sqrt{a}$ and is bounded from below by 0 . Since the Ricci curvature of $M$ is bounded from below and since $M$ is complete and space-like, we can apply the Generalized Maximum Principle (Theorem 2.1) to the function $F$. For any given positive number $\varepsilon$, there exists a point $p$ at which $F$ satisfies (3.16). Consequently the following relationship

$$
\frac{1}{2} F(p)^{4} \Delta|\mu|^{2}(p)<3 \varepsilon^{2}+F(p) \varepsilon
$$

can be derived by the simple and direct calculations. For any convergent sequence $\left\{\varepsilon_{m}\right\}$ such that $\varepsilon_{m} \rightarrow 0(m \rightarrow \infty)$ and $\varepsilon_{m}>0$, there exists a point sequence $\left\{p_{m}\right\}$ such that $\left\{F\left(p_{m}\right)\right\}$ converges to $F_{0}=\inf F$ by (3.16). On the other hand, it follows from (5.3) that we have

$$
\frac{1}{2} F\left(p_{m}\right)^{4} \Delta|\mu|^{2}\left(p_{m}\right)<3 \boldsymbol{\varepsilon}_{m}{ }^{2}+F\left(p_{m}\right) \boldsymbol{\varepsilon}_{m} .
$$

The right hand side of (5.4) converges to 0 , because the function $F$ is bounded. Accordingly, for any positive number $\varepsilon$ there exists a sufficiently large integer $m_{0}$ for which we have

$$
F\left(p_{m}\right)^{4} \Delta|\mu|^{2}\left(p_{m}\right)<\varepsilon \text { for } m>m_{0} .
$$

Since it is seen by Proposition 4.1 that the function $|\mu|^{2}$ is bounded, the infimum $F_{0}$ of the function $F$ satisfies $F_{0} \neq 0$ and hence the inequality (5.5) yields that $\lim \sup \Delta|\mu|^{2}\left(p_{m}\right) \leqq 0$. This means that the supremum of $|\mu|^{2}$ is equal to 0 by (5.2), because $\delta$ is the positive constant. So we obtain $\mu=0$, i.e., $M$ is pseudo-umbilic, which yields that the equality of (3.14) in Proposition 3.2 holds. Then the equalities of all inequalities in Section 3 have to hold. Consequently, from (3.4) and (3.13) it is seen that we have

$$
h_{i j k}^{\alpha}=0
$$

for any $\imath, \jmath, k$ and $\alpha$. Also from (3.2) and (3.11) it follows that we get

$$
H^{\alpha} H^{\beta}=H^{\beta} H^{\alpha}
$$

for any $\alpha$ and $\beta$. The equations imply that all of $H^{\alpha}$ are simultaneously diagonalizable and the normal connection in the normal bundle of $M$ is flat. Hence we can choose a suitable basis $\left\{e_{\imath}\right\}$ such that

$$
h_{i j}^{\alpha}=\lambda_{i}^{\alpha} \delta_{i j}
$$

for any $\imath, \jmath$ and $\alpha$. The submanifold $M$ is said to be isoparametric [9] if the normal connection is flat and the charactristic polynomial of the shape operator $A_{\xi}$ for any local parallel normal field $\xi$ is constant over the domain.

LEMMA 5.1. $M$ is isoparametric. 
Proof. Since the normal connection is flat, it is seen that there exist locally $p$ mutually orthogonal unit normal vector fields which are parallel in the normal bundle. So we can choose a suitable parallel basis $\left\{e_{\alpha}\right\}$ and then we have $\omega_{\alpha \beta}=0$. Hence, since we have

$$
\sum h_{i j k}^{\alpha} \omega_{k}=d h_{i j}^{\alpha}-\sum h_{k j}^{\alpha} \omega_{k i}-\sum h_{i k}^{\alpha} \omega_{k j}+\sum h_{i j}^{\beta} \omega_{\beta \alpha},
$$

setting $i=\jmath$ in the above equation and using (5.6) we get $d h_{\imath \imath}^{\alpha}=0$. Hence $h_{i i}^{\alpha}$ is constant and $M$ is isoparametric.

LEMMA 5.2. $M$ is of non-positive curvature.

Proof. Suppose first that there exist indices $\imath, \jmath$ and $\alpha$ such that $h_{\imath \imath}^{\alpha} \neq h_{\jmath \jmath}^{\alpha}$. From the equation (5.9) we get

$$
\sum h_{k j}^{\alpha} \omega_{k i}+\sum h_{i k}^{\alpha} \omega_{k j}=\left(h_{i i}^{\alpha}-h_{j j}^{\alpha}\right) \omega_{i \jmath}=0,
$$

from which it follows that $\omega_{i j}=0$. For any index $\imath$ we denote by $[i]$ the set of indices $k$ such that $h_{k k}^{\alpha}=h_{i i}^{\alpha}$. Under this notation the above property shows

$$
\omega_{i k}=0 \quad \text { for any } k \notin[i] \text {. }
$$

Accordingly, we obtain

$$
\sum \omega_{i k} \wedge \omega_{k J}=0 .
$$

In fact, the left hand side of the above equation can be regarded as

$$
\sum \omega_{i k} \wedge \omega_{k \jmath}=\sum_{k \in[i]} \omega_{i k} \wedge \omega_{k j}+\sum_{k \in[j]} \omega_{i k} \wedge \omega_{k \jmath}+\sum_{k \notin[\imath] \cup[j]} \omega_{i k} \wedge \omega_{k \jmath},
$$

each term of which vanishes identically, because of (5.10). Thus, from the structure equation

$$
d \omega_{i j}+\sum \omega_{i k} \wedge \omega_{k J}=-\frac{1}{2} \Sigma R_{k \imath \jmath l} \omega_{k} \wedge \omega_{l},
$$

we obtain

$$
R_{\imath \jmath j i}=c-\sum_{\beta} \lambda_{i}^{\beta} \lambda_{j}^{\beta}=0 .
$$

Next, suppose that $h_{i i}^{\alpha}=h_{\jmath \jmath}^{\alpha}$ for distinct indices $\imath$ and $\jmath$ and for any $\alpha$. Then the Gauss equation implies

$$
R_{\imath j j i}=c-\sum_{\alpha}\left(h_{i i}^{\alpha}\right)^{2}=c-\sum_{\alpha}\left(\lambda_{\imath}^{\alpha}\right)^{2}=c-H^{2}-\sum_{\alpha \neq 4}\left(\lambda_{\imath}^{\alpha}\right)^{2} \leqq 0,
$$

because of $H^{2}-c \geqq 0$.

Thus $M$ is of non-positive curvature.

Proof of Theorem 2. First of all, we notice that $M$ is not totally umbilic under the condition (5.1). In fact, suppose that $M$ is totally umbilic. The equation (3.6) means that $M$ is totally umbilic if and only if $S=n H^{2}$, from 
which combining with the second equation of (5.1) it follows that we have $H^{2}=c=0$. So $M$ is totally geodesic and it satisfies $\operatorname{Ric}(M)=0$. On the other hand, by the first equation of $(5.1)$, we get $\operatorname{Ric}(M)<0$, a contradiction.

Now we consider an $n$-dimensional space-like submanifold $M$ of $\boldsymbol{R}_{p}^{n+p}$. By a theorem due to Koike [6] and Lemmas 5.1 and 5.2 it is seen that $M$ is locally congruent to the product submanifold

$$
H^{n_{1}}\left(c_{1}\right) \times \cdots \times H^{n_{q}}\left(c_{q}\right) \times \boldsymbol{R}^{m}
$$

of $\boldsymbol{R}_{q}^{n+q}$ whose mean curvature vector is parallel in the normal bundle of $M$ in $\boldsymbol{R}_{q}^{n+q}$, where $\sum_{r=1}^{q} n_{r}+m=n, q \geqq 0, m \geqq 0$ and $\boldsymbol{R}_{q}^{n+q}$ is a totally geodesic submanifold of $\boldsymbol{R}_{p}^{n+p}$. Then $M$ can be naturally regarded as the space-like submanifold of $\boldsymbol{R}_{p}^{n+p}$.

The condition for the codimension is next given. For the purpose the squared norm $S$ of the second fundamental form and the mean curvature $H$ of $M$ in $\boldsymbol{R}_{q}^{n+q}$ and hence in $\boldsymbol{R}_{p}^{n+p}$ are calculated. In fact, the product manifold is constructed as follows: Without loss of generality, an $(n+q)$-dimensional semiEuclidean space $\boldsymbol{R}_{q}^{n+q}$ of index $q \geqq 0$ can be first regarded as a product manifold of

$$
\boldsymbol{R}_{1}^{n_{1}+1} \times \cdots \times \boldsymbol{R}_{1}^{n_{q}+1} \times \boldsymbol{R}^{m},
$$

where $\sum_{r=1}^{q} n_{r}+m=n$. With respect to the standard orthonormal basis of $\boldsymbol{R}_{q}^{n+q}$ a class of space-like submanifolds

$$
H^{n_{1}}\left(c_{1}\right) \times \cdots \times H^{n_{q}}\left(c_{q}\right) \times \boldsymbol{R}^{m}
$$

of $\boldsymbol{R}_{q}^{n+q}$ is defined as the Pythagorean product

$$
\begin{aligned}
& H^{n_{1}}\left(c_{1}\right) \times \cdots \times H^{n_{q}}\left(c_{q}\right) \times \boldsymbol{R}^{m} \\
& \quad=\left\{\left(x_{1}, \cdots, x_{q+1}\right) \in \boldsymbol{R}_{q}^{n+q}=\boldsymbol{R}_{1}^{n_{1}+1} \times \cdots \times \boldsymbol{R}_{1}^{n^{q}+1} \times \boldsymbol{R}^{m}:\left|x_{r}\right|^{2}=-\frac{1}{c_{r}}>0\right\},
\end{aligned}
$$

where $r=1, \cdots, q$ and || denotes the norm defined by the product on the Minkowski space $\boldsymbol{R}_{1}^{k+1}$ which is given by $\langle x, x\rangle=-\left(x_{0}\right)^{2}+\sum_{j=1}^{k}\left(x_{\jmath}\right)^{2}$. The mean curvature vector $\boldsymbol{h}$ of $M$ in $\boldsymbol{R}_{q}^{n+q}$ and hence in $\boldsymbol{R}_{p}^{n+p}$ is given by

$$
\boldsymbol{h}=-\frac{1}{n}\left(n_{1} c_{1} x_{1}+\cdots+n_{q} c_{q} x_{q}\right)
$$

at $x=\left(x_{1}, \cdots, x_{q+1}\right) \in M$, which is parallel in the normal bundle of $M$. So, the squared norm $S$ of the second fundamental form and the mean curvature $H$ of $M$ in $\boldsymbol{R}_{p}^{n+p}$ are given by

$$
S=-\sum_{r=1}^{q} n_{r} c_{r}, \quad n^{2} H^{2}=-\sum_{r=1}^{q} n_{r}^{2} c_{r},
$$

which yields

$$
S-p n H^{2}=\frac{1}{n} \sum_{r=1}^{q} n_{r}\left(p n_{r}-n\right) c_{r}=0 .
$$


Suppose that $p \leqq 3$. Then we see $\operatorname{Ric}(M) \leqq \delta_{1}<0$ by (5.1). Since $M$ is 3 dimensional and it is congruent to the product submanifold (5.11), the negative definiteness of the Ricci curvature means that $M$ is totally umbilic, a contradiction. We next suppose $p \geqq 4$. This means that $M$ is totally umbilic by (5.12), a contradiction.

Hence the case of $c=0$ can not occur.

Suppose next that $c<0$. By means of Koike's theorem and Lemmas 5.1 and 5.2 again, $M$ is locally congruent to the product submanifold $H^{n_{1}}\left(c_{1}\right) \times \cdots \times$ $H^{n^{+}+1}\left(c_{q+1}\right)$ in $H_{q}^{n+q}\left(c^{\prime}\right)$, where $\sum_{r=1}^{q+1} n_{r}=n, q \geqq 0$, and $\sum_{r=1}^{q+1}\left(1 / c_{r}\right)=\left(1 / c^{\prime}\right) \geqq(1 / c)$, and $H_{q}^{n+q}\left(c^{\prime}\right)$ is a totally umbilic submanifold of $H_{p}^{n+p}(c)$.

We investigate the relation between the mean curvature $H$ and the squared norm $S$ of $M$ in $H_{p}^{n+p}(c)$. We consider an $n$-dimensional space-like submanifold with parallel mean curvature vector of $H_{q}^{n+q}\left(c^{\prime}\right)$. Without loss of generality, an $(n+q+1)$-dimensional indefinite Euclidean space $\boldsymbol{R}_{q+1}^{n+q+1}$ of index $(q+1)$ can be regarded as a product manifold of

$$
\boldsymbol{R}_{1}^{n_{1}+1} \times \cdots \times \boldsymbol{R}_{1}^{n_{q+1}+1}
$$

where $\sum_{r=1}^{q+1} n_{r}=n$. With respect to the standard orthonormal basis of $\boldsymbol{R}_{q+1}^{n+q+1}$ a class of space-like submanifolds

$$
H^{n_{1}}\left(c_{1}\right) \times \cdots \times H^{n_{q+1}}\left(c_{q+1}\right)
$$

of $\boldsymbol{R}_{q+1}^{n+q+1}$ is defined as the Pythagorean product

$$
\begin{aligned}
& H^{n_{1}}\left(c_{1}\right) \times \cdots \times H^{n_{q+1}}\left(c_{q+1}\right) \\
& \quad=\left\{\left(x_{1}, \cdots, x_{q+1}\right) \in \boldsymbol{R}_{q+1}^{n+q+1}=\boldsymbol{R}_{1}^{n_{1}+1} \times \cdots \times \boldsymbol{R}_{1}^{n_{q+1}+1}:\left|x_{r}\right|^{2}=-\frac{1}{c_{r}}>0\right\},
\end{aligned}
$$

where $r=1, \cdots, q+1$. The mean curvature vector $\boldsymbol{h}^{\prime}$ of $M$ in $H_{q}^{n+q}\left(c^{\prime}\right)$ is given by

$$
\boldsymbol{h}=-\frac{1}{n} \sum_{r=1}^{q+1}\left(n_{r} c_{r} x_{r}\right)-c^{\prime} x
$$

at $x=\left(x_{1}, \cdots, x_{q+1}\right) \in M$, which is parallel in the normal bundle of $M$ in $H_{q}^{n+q}\left(c^{\prime}\right)$. So the mean curvature $H^{\prime}$ and the squared norm $S^{\prime}$ of the second fundamental form of $M$ in $H_{q}^{n+q}\left(c^{\prime}\right)$ are given by

$$
n^{2} H^{\prime 2}=n^{2} c^{\prime}-\sum_{r=1}^{q+1} n_{r}^{2} c_{r}, \quad S^{\prime}=n c^{\prime}-\sum_{r=1}^{q+1} n_{r} c_{r} .
$$

For the mean curvature vector $\boldsymbol{h}^{\prime}$ of $M$ in $H_{q}^{n+q}\left(c^{\prime}\right)$ the mean curvature vector $\boldsymbol{h}$ of $M$ in $H_{p}^{n+p}(c)$ is given by $\boldsymbol{h}=\boldsymbol{h}^{\prime}+\boldsymbol{h}^{\prime \prime}$, where $\boldsymbol{h}^{\prime \prime}$ is the mean curvature vector of $H_{q}^{n+q}\left(c^{\prime}\right)$ in $H_{p}^{n+p}(c)$. Consequently, by using (5.14) the mean curvature $H$ and the squared norm $S$ of $M$ in $H_{p}^{n+p}(c)$ are given by

$$
n^{2} H^{2}=n^{2} c^{\prime}-\sum_{r=1}^{q+1} n_{r}{ }^{2} c_{r}+(p-q)^{2}\left(c-c^{\prime}\right),
$$




$$
S=n c-\sum_{r=1}^{q+1} n_{r} c_{r}+(p-q)\left(c-c^{\prime}\right),
$$

from which it follows that we have

$$
\begin{aligned}
S- & \left\{n p H^{2}-n(p-1) c\right\} \\
= & \frac{1}{n} \sum_{r=1}^{q+1} n_{r}\left(p n_{r}-n\right) c_{r}+\left\{(p-q)+p n-\frac{p}{n}(p-q)^{2}\right\}\left(c-c^{\prime}\right) .
\end{aligned}
$$

Suppose that $p \leqq 3$. Then we see $\operatorname{Ric}(M)<0$ by (5.1). Since $M$ is congruent to the product manifold (5.13) and it is 3-dimensional, the negative definiteness of the Ricci curvature means that $M$ is totally umbilic, a contradiction. Accordingly, we obtain $p \geqq 4$. On the other hand, $q$ must be less than 3 , because of $n=3$. In order to check whether or not these situations occur, it is divided into three cases: $q=0,1$ and 2 .

First we consider the case $q=0$. Then $M$ is totally umbilic, a contradiction.

Next we consider the case $q=1$. If $p \geqq 5$, then the first term of the right hand side in (5.15) is negative and the second one is of non-positive. This also leads a contradiction. So we have $p=4$ and $c_{1}$ and $c_{2}$ are determined by constant curvatures $c$ and $c^{\prime}$, because of $1 / c_{1}+1 / c_{2}=1 / c^{\prime}$.

The case $q=2$. If $p \geqq 6$, then the first term of the right hand side in (5.15) is negative and the second one is of non-positive. Accordingly this case can not occur. So we have $p=4$ or $p=5$.

This completes the proof.

Remark 5.1. A product manifold $H^{1}\left(c_{1}\right) \times H^{1}\left(c_{2}\right) \times H^{1}\left(c_{3}\right)$ is a canonical spacelike submanifold with parallel mean curvature vector of $H_{3}^{6}(c)$ and it satisfies $\operatorname{Ric}(M)=0$ and $S=9 H^{2}-6 c$. This means that the estimate of the Ricci curvature is best possible.

Remark 5.2. In the case of $p=1$, two conditions in (5.1) are equivalent with each other.

\section{REFERENCES}

[1] R. Aiyama And Q. M. Cheng, Complete space-like hypersurfaces in a Lorentz space form of dimension 4, To appear in Kodai Math. J..

[2] E. CAlABI, Examples of Bernstein problems for some nonlinear equations, Proc. Pure Appl. Math. 15 (1970), 223-230.

[3] Q. M. Cheng, Complete space-like submanifolds in de Sitter space with parallel mean curvature vector, Math. Z. 206 (1991), 333-339.

[4] S. Y. Cheng And S.T. YAU, Maximal space-like hypersurfaces in the LorentzMinkowski spaces, Ann. of Math. 104 (1976), 407-419.

[5] T. IsHiHARA, Maximal spacelike submanifolds of a pseudo Riemannian space of constant mean curvature, Michigan Math. J. 35 (1988), 345-352.

[6] N. Kolke, Proper isoparametric semi-Riemannian submanifolds in a semi-Riemannian space-form, Tsukuba J. Math. 13 (1989), 131-146. 
[7] S. Nishikawa, On maximal spacelike hypersurfaces in a Lorentzian manifold, Nagoya Math. J. 95 (1984), 117-124.

[8] H. OMORI, Isometric immersions of Riemannian manifolds, J. Math. Soc. Japan 19 (1967), 205-214.

[9] C.L. TERnG, Isoparametric submanifolds and their coxeter groups, J. Differential Geometry 21 (1985), 79-107.

[10] A.E. TREIBERgS, Entire hypersurfaces of constant mean curvature in Minkowski 3-space, Invent. Math. 66 (1982), 39-56.

[11] S. T. YAU, Harmonic functions on complete Riemannian manifolds, Comm. Pure and Appl. Math. 28 (1975), 201-208.

INSTITUTE OF MATHEMATICS,

UNIVERSITY OF TSUKUba, 305 IBARAKI,

JAPAN 